\title{
Effect of aging and anthropometric measurements on nerve conduction properties - A Review
}

\author{
Jagga, M., Lehri, A. \& Verma, S.K. \\ Research Scholar, Dept of Sports Science, Punjabi University, Patiala-147001, Punjab \\ Assistant Professor, Dept of Sports Science, Punjabi University, Patiala-147001, Punjab \\ Professor, Dept of Sports Science, Punjabi University, Patiala-147001, Punjab
}

\section{Abstract}

Aging is a process that is often accompanied by physiological changes. These physiological changes include slowing in muscle contractility, alteration in muscle metabolism and neuromuscular junction, and reduction in nerve conduction velocity (NCV). Age has been widely accepted to have an influence on nerve velocity. It is well established that there is a decline in muscular performance with advancing age. Nerve conduction studies (NCS) are the most sensitive and reproducible measure of peripheral nerve functions. Nerve conduction studies (NCS) are performed to diagnose the disorders of the peripheral nervous system. These enable the clinicians to differentiate the two major groups of peripheral diseases: demyelination and axonal degeneration. These also help in localizing the site of the lesions. Majority of the available literature mentioning effects of age and anthropometric factors on nerve conduction measures have been referred in the present review. Various studies referred to are based on the data on healthy subjects. The references were further supplemented by systematic search on pubmed, google and Pedro with keywords age, temperature, height and nerve conduction. Several specific studies that had been dealt on anthropometric measures and nerve conduction on normal subjects were also included in this review. A total of fifty five research studies dealing with age, anthropometric measurements and nerve conduction properties have been found in the literature reviewed up to 2011.

KEY WORDS: nerve conduction velocity, anthropometric, Nervous System

\section{Introduction}

Aging describes changes that occur with advancing age. Normally physiological capacity of various systems attains a maximum level in $3^{\text {rd }}$ decade of life between the late teens and thirty years of age. After 35 years there occurs a decline in physiologic and performance measures. Different systems change at different rates in different people. Chronological age does not necessarily correlate with biological age. For example, an 85 year old may be active and independent where as 65 year old may have many problems. With age there is decline in energy, faculties and tissues. Visible signs of aging include graying and coarseness of hair, loss of elasticity and dryness of the skin.

Age- related changes in musculoskeletal system

Age related changes in muscle system are directly related to limited mobility and increase of falls in the elderly. Aging is associated with decrease in total muscle cross-sectioned area amounting to approx $40 \%$ between the ages of 20 and 80 years. Cummingham et.al in 1989 and 1982 conducted study on young and elderly men and found that this reduction in muscle cross-sectioned area is accompanied by increase in non contractive structures such as fat and connective tissue. Thus, the girth or volume of the muscle band on 
anthropometric measurements may not show the actual reduction in excitable muscle mass. The total number of muscle fibers is significantly reduced with age beginning at about 25 years and progressing at an accelerated rate thereafter.

Grimby et al (1984), Lexell et al (1988) and Porter et al (1995) have concluded that type II fibers size of vastus lateralis, tibialis anterior and biceps brachii decreases with increasing age, whereas type I fiber size does not change with age. The most basic age related change in muscle morphology at the level of the Motor Unit (MU) is a decrease in the number of total Motor Units. Tomlinson et al (1977) in their study found that loss in MU number is approximately $1 \%$ of the total number per annum, beginning in the third decade of life and increasing rapidly in rate beyond the age of 60. Age-related decreases in strength have been well documented. A variety of limb muscles have been tested during isometric strength tasks in young, middle-aged, and older men and women. In general, results of several studies demonstrate that reductions in isometric and dynamic voluntary strength become substantial by the seventh decade of life, and may accelerate thereafter. Healthy men and women in their seventh and eighth decades of life demonstrates average reductions of $20-40 \%$ in maximal isometric strength in various muscles, when compared to young adults (McDonagh et al, 1984, Young et al, 1984, Davies et al, 1986, Kallman et al, 1990). These age-related reductions are relatively similar for both sexes and for proximal and distal muscles of various sizes, in both upper and lower limbs. Muscular strength is reported to be highest between the ages of 20 and 30 2 years. Though, there is progressive decline in maximum strength and which entirely leads to functional loss in activities of daily living.

Age-related changes in nervous system:

Research reports indicate that as brain ages or as people get older, there occurs a decrease in brain weight and brain volume, widening of the grooves on the surface of the brain and enlargement of the ventricular system. The decrease in brain weight and brain volume is probably due to the loss of neurons and extra cellular fluid. Man may have a $20 \%$ reduction in brain weight between the ages of forty -five and eighty -five and lose thirty to fifty thousand neurons a day from the brain and nervous system as they age. Enlargement of the ventricular system may be probably due to loss of cells surrounding the ventricles. In the first studies of the effects of age on the human Cortex, Brody (1955, 1970) concluded that as many as 50\% of neurons are lost with age. Other investigators concurred with this conclusion and it was not until the 1980s that Haug and Terry and their colleagues produced contrary evidence. Haug and his colleagues (Haug, 1984, 1985; Haug et al., 1984) showed that many of the early reports of loss of cortical neurons with age could be attributed to the fact that upon fixation the brains of younger individuals shrink more than those of older ones, which have more myelin. Consequently, when sections of cortical tissue are examined, the young brains show higher neuronal packing densities than those of older individuals. After making corrections for this differential shrinkage, Haug and his colleagues concluded there is no significant loss of neurons from the human cerebral cortex 
with age. Terry et al (1987) reached a similar conclusion and suggested that much of the neuronal loss recorded in earlier studies could be attributed to brains of some individuals with undiagnosed Alzheimer's disease being included among the older brains that were being evaluated and considered to be normal. In Alzheimer's disease there is a significant neuronal loss from cerebral cortex. According to Peter (2002) there is no evidence for a significant loss of cortical neurons during normal aging on the cerebral hemispheres, however there is widespread damage to the myelin sheaths that ensheath their axons, as evidenced by structurally altered myelin sheaths observed in electron microscopic preparations. Although there is no extensive loss of cortical neurons in normal aging, neurons do lose dendritic branches and spines, and there are alterations in the levels of some neurotransmitters and their receptors.

Aging is a process that is often accompanied by physiological changes. These physiological changes include slowing in muscle contractility, alteration in muscle metabolism and neuromuscular junction, and reduction in nerve conduction velocity (NCV). Age has been widely accepted to have an influence on nerve velocity. It is well established that there is a decline in muscular performance with advancing age. Common observations of muscle wasting in the elderly, particularly of proximal limb muscles as well as the small muscles of the hand are reported with aging. Undoubtedly many extraneous factors may contribute to neuromuscular disease in the elderly, of which the most important is probably malnutrition, disuse, circulatory impairment, etc.

Nerve conduction studies (NCS) are the most sensitive and reproducible measure of peripheral nerve functions. Nerve conduction studies (NCS) are performed to diagnose the disorders of the peripheral nervous system. These enable the clinicians to differentiate the two major groups of peripheral diseases: demyelination and axonal degeneration. These also help in localizing the site of the lesions. NCS consist primarily of the assessment of three types of nerves: motor, sensory and mixed. Motor NCS include the assessment of the compound muscle action potential (CMAP), whereas sensory NCS include the assessment of the sensory nerve action potentials (SNAP) of the accessible peripheral nerves in the upper and lower limbs. The median, ulnar, radial, common peroneal, tibial and the sural nerves are the commonly examined nerves. The commonly measured parameters of the CMAP include latency, amplitude, duration, conduction velocity and late response, e.g., F-waves. Similarly, for SNAP, latency, amplitude and conduction velocity are routinely measured. Nerve conduction parameters may be affected by anthropometric factors like age, sex, height, weight and BMI. As it has been reported that significant slowing of conduction velocities and sensory latencies occur with increasing age and more height. It was claimed further that the conduction velocity is $6 \mathrm{~m} / \mathrm{sec}$ faster in females. Flack et al (1994) reported in their study that the conduction velocity in a newborn is approximately $50 \%$ of adult values and progressively increase, reaching the adult value at the age of 
three. Later in adulthood, the nerve velocity decreases with age and this is more pronounced in the lower than in the upper limbs. Rivner et al (2001) studied that the correlation of NCV with height was stronger than with age. Regression equations using both factors account for $12-27 \%$ of the variance. Responses were seen in the majority of patients aged 70 years and older, but the percentage of normals who had no response increased with advancing age. Age was strongly inversely correlated with the amplitudes of both sensory and motor responses, accounting for $7-16 \%$ of the variance. Regression equations using both height and age improved this correlation and accounted for $7-22 \%$ of the variance.

\section{Materials \& Methods}

Majority of the available literature mentioning effects of age and anthropometric factors on nerve conduction measures has been referred. Various studies referred to are based on the data on healthy subjects. The references were further supplemented by systematic search on pubmed, google and Pedro with keywords age, temperature, height and nerve conduction. Several specific studies that had been dealt on anthropometric measures and nerve conduction on normal subjects were also included in this review. The search was further refined by restricting search to English language publications and to papers that included specified epidemiological terms. Irrelevant references were eliminated in this review by the researchers. To test the adequacy of our search strategy the outcome for the period up to 2011 was included in this review.

\section{Results}

A total of fifty five research studies dealing with age, anthropometric measurements and nerve conduction properties have been found in the literature reviewed up to 2011.

Saeed (2008) reported that sural nerve conduction velocity had significant inverse correlation with age. It had also weak inverse relation with height, while sural sensory latency had insignificant direct relationship with age and height. Both neurological parameters have been reported to vary more with change in age than the change in height so necessitating adjustments for height and age must be considered while developing normal standard values in (40-70 years) age range of the subjects. According to him, more research on wider data base is required to develop norms and standards in this context. In the age range of 40-70 years there is no gender specific difference in the conduction measures of the sural nerve. Mohamed in 2007 revealed that The mean velocities for the median and ulnar nerves, both motor and sensory, were $54.71 \pm 5.69 \mathrm{~m} / \mathrm{s}$ (motor) and $54.04 \pm 7.02 \mathrm{~m} / \mathrm{s}$ for the median nerve and $60.57 \pm 5.00 \mathrm{~m} / \mathrm{s}$ (motor) and 52.92 \pm 5.89 $\mathrm{m} / \mathrm{s}$ for the ulnar nerve. The mean velocity for the sural nerve was $47.97 \pm 4.48 \mathrm{~m} / \mathrm{s}$. Only median motor conduction velocity showed a significant reduction with increasing age $(p=0.000)$ $\&$ concluded that there is no significant effect of age on nerve conduction velocities except for median motor conduction velocity. Falco et al in 1992 reported that age had a statistically significant but low strength effect on all ulnar nerve conduction velocities and distal latencies as well as the distal sensory amplitudes of all three nerves. They also explored that gender had a greater effect than age on these 
parameters as well as on median sensory distal latency. Other median motor and sensory conduction parameters along with radial sensory distal latency were not significantly related to age or gender. Dilip in 2010 studied that gender has definite effects on NCS variables. Males had higher CMAP amplitude, longer latencies and duration. SNAP latencies and duration were longer in males whereas amplitude was higher in female whereas Robinson et al (1993) concluded that most gender differences in nerve conduction velocity can be largely explained by height, whereas amplitude differences persist despite correction for height, temperature, and age.

Diana et al in 1992 found that height was negatively associated with sensory amplitude in all nerves tested $(P<0.001)$, and positively associated with median and ulnar sensory distal latencies $(P<0.01)$ and sural latency $(P<0.001)$. She also reported that index finger circumference was negatively associated with median and ulnar sensory amplitudes $(P<0.05)$ and sex, in isolation from highly correlated anthropometric factors such as height, was not a significant predictor of median or ulnar nerve conduction measures. Michael et al in 2001 reported the correlation of NCV with height was stronger than with age. Regression equations using both factors account for $12-27 \%$ of the variance. He had seen responses in the majority of patients aged 70 years and older, $\mathrm{He}$ also found that percentage of normal that had no response increased with advancing age and age was strongly inversely correlated with the amplitudes of both sensory and motor responses, accounting for $7-16 \%$ of the variance. Regression equations using both height and age improved this correlation, accounting for $7-22 \%$ of the variance. Thakur et al (2010) explored that the height showed a significant correlation with the nerve conduction parameters of most of the motor nerves and a few sensory nerves. They also concluded that diagnoses which were made from the nerve conduction data without making corrections for height may be invalid in patients who are taller and shorter than average individuals. This must be also considered while developing standard/reference normative data for different nerves.

David et al (1989) investigated that temperature has a profound inverse effect on SNAP latency and must be accounted for during clinical electrophysiological examinations. Conversely, temperature has a direct effect on SNAP amplitude and must also be considered during EMG examinations.

According to Rabben (1995) values of $\mathrm{CV}$ along the segment wrist to elbow in newborns (CVo) and after maturation (CVmax) were found to be significantly higher than those along the distal fingers to wrist segment of both median and ulnar nerves $(p<0.001)$. The main reason for this is probably temperature difference between these two segments. Secondly Smaller variations of CV values found in different nerves along the same nerve segment can be caused by measurement or other technical error. Besides, in case of infants it is also very important that age-groups are identical.

Ralph (1999) stated that there is no significant difference between blacks and whites in normal nerve conduction study findings in healthy adults. Tong et al 
(2004) reported the rate of change over time was not affected by hand (dominant versus non-dominant hand), gender, age, or BMI at baseline. The rate of change seen with some of the median nerve parameters was significantly greater than that with the ulnar nerve.

\section{Discussion}

The results of various literatures describe the statistical significance of associations.

\section{Age:}

The conclusion of 55 research studies revealed that nerve conduction velocity decreases with age. The decrease in nerve conduction velocity may be because of decreased number of nerve fiber, a reduction in fiber diameter and changes in the fiber membrane The study done by Dorfman and Bosley of 30 normal subjects (15 young and 15 older adults) estimated a decrease of $0.16 \mathrm{~m} / \mathrm{s}$ per year of age, that by Stetson (1982) of 105 normal workers (excluding workers on jobs thought to involve repetitive or forceful hand exertions) estimated a decrease of $0.13 \mathrm{~m} / \mathrm{s}$ per year, and that by Letz and Gerr of over 4000 veterans estimated a decrease of $0.13 \mathrm{~m} / \mathrm{s}$ per year. The $1.3 \mathrm{~m} / \mathrm{s}$ decrease in median sensory distal conduction velocity and the $0.8 \mathrm{~m} / \mathrm{s}$ decrease in motor conduction velocity per decade of aging were found in study done by Diana et al. But Mitchael et al in there study found a less correlation between age and nerve conduction velocity. In an another study done by Mohamed et al (2007) it was also concluded that there is not any significant effect of age on nerve conduction velocities except for median nerve.

Like nerve conduction velocity responses amplitude and distal latencies show a stronger inverse correlation with age. The distribution of amplitude values in the young is much wider than in elderly. Fewer higher amplitude values are seen after the age of 60 years. A study done by Diana et al showed an average loss of about $5 \mathrm{IJ} . \mathrm{V}$ per decade for wristdigit I1 sensory amplitude. This was somewhat larger than the $1.5 \mathrm{IJ} . \mathrm{V}$ per decade loss in digit 111- wrist sensory amplitude reported by Buchthal et al (1974) .6 or the 3.8 IJ.V per decade loss in wrist-di it 111 sensory amplitude reported by Tackmann. The reduced nerve amplitude is best related to loss of axons. The increase in oedema found in elderly along with increased skin resistance may also play a role in the reduction of amplitude seen with ageing.

\section{Temperature}

Over relatively wide temperature ranges, motor and sensory nerve conduction velocities have a positive linear relationship with body temperature. With cooling, motor and sensory amplitudes increase and conduction values decrease. Cooling is thought to affect muscle and nerve membrane function, particularly the sodium ion channel. A direct relationship was observed between the distal sensory amplitude of sural sensory nerve action potential and the temperature of the leg. Henriksen (1956) studied the temperature effects from $12^{\circ}$ to $40^{\circ} \mathrm{C}$ on the motor conduction of human nerves. He found that between $29^{\circ}$ and $38^{\circ} \mathrm{C}$ the motor conduction velocity decreased $2.4 \mathrm{~m} / \mathrm{sec}$ for each $1{ }^{\circ} \mathrm{C}$ decrease in temperature. Similar decreases in neural conduction as a function of a $1{ }^{\circ} \mathrm{C}$ decrease in limb temperature were reported by Buchthal and Rosenfalck (1971) $(2.0 \mathrm{~m} / \mathrm{sec})$, McLeod $(2.6 \mathrm{~m} / \mathrm{sec})$, deJesus et al $(2.1$ 
$\mathrm{m} / \mathrm{sec})$, Ludin and Beyeler (1977) (1.51 $\mathrm{m} / \mathrm{sec}$ ), and Bolton et al (1981) (2.1 $\mathrm{m} / \mathrm{sec}$ ). Ludin and Beyeler (1977) have repotted that between $22^{\circ}$ and $26^{\circ} \mathrm{C}$ the amplitude of SNAPs decreased with lowering temperatures of the limb. Oh (1982) and Ludin and Beyeler (1977) attributed an increase in SNAP amplitude with a concomitant increase in limb temperature to a smaller temporal dispersion of the SNAPs for different fibers and to a sequential shortening of the SNAP spike. As temporal dispersion diminishes with an increase in limb temperature, the SNAP amplitude increases. Bolton et al (1981) however, found that between limb temperatures of $21^{\circ}$ to $31^{\circ} \mathrm{C}$, the amplitude of the SNAP showed a progressive linear increase with decreasing temperature. This increase in SNAP amplitude with decreasing temperatures may be explained by a decrease in temporal dispersion rather than a real increase in height (amplitude) of the SNAP.

\section{Height}

The negative relationship between height and sensory amplitudes was a consistent finding in sensory nerves tested in most of the studies. While controlling for age and surface temperature, height explained much of the sensory amplitude variation (partial R2 in 0.20 to 0.25 ranges). Our results duplicated those of others who have found a strong negative correlation between height and either sural or peroneal conduction velocity. Soudmand et al (1982) studied the effect of height on nerve conduction velocity (NCV) on 41 normal subjects and found that peroneal and sural NCV correlated inversely with height and with estimated axonal length, whereas median motor and sensory NCV failed to show any significant relationship to height. Thakur (2011) in there study on 34 healthy subjects also supported result of various studies that height has a negative correlation with the sensory nerve action potential amplitudes.

Race

The findings of various research studies show that there is no significant difference between race and nerve conduction. In a comparative study done by Ralph et al on 50 Blacks and 50 Whites it was concluded that there was no significant difference between blacks and whites in normal nerve conduction study findings in healthy adults.

\section{Gender difference}

The results of various literatures revealed that there is no significant difference between gender and median or ulnar nerve conduction measures in healthy subjects. The results was supported by study done by Stetson (1982) on 105 asymptomatic healthy adults in which it was found that there is no association between sex and median or ulnar nerve conduction measures. For the sural nerve, using a model which also included age, midcalf temperature, and height, women were found to have significantly smaller amplitude and slower conduction velocity then men. The most plausible hypothesis is that both sex and sural nerve conduction and amplitude are correlated with an anatomical or physiological factor which we did not measure. Our results were also supported by study done by Greathouse (1989) on 22 healthy subjects which also explains that there is no significance difference between gender and nerve conduction 
measures. But Thakur $(2010,2011)$ in a gender based study on 34 healthy subjects concluded that gender has definite effects on NCS variables. Males had higher CMAP amplitude, longer latencies and duration. Sensory nerve action potential latencies and duration were longer in males whereas amplitude was higher in females. Without adjustment for these factors, the sensitivity and specificity of NCS will decrease when using the same reference data in patients with different gender.

\section{BMI:}

Our findings explore that the sural sensory conduction velocity had a non significant negative relationship with BMI. The inverse correlation of sensory conduction velocity and direct relation of sensory latency with BMI indicated the sole effect of height among these relationships. So it is quite obvious that weight and obesity do not have any significant impact on nerve conduction parameters. The findings were supported by study done by Stetson (1982) on 105 asymptomatic healthy adults.

\section{References}

Akataki, K., Mita, K., Watakabe, M., Ito, K. 2002. Age-Related Change in Motor Unit Activation Strategy In force Production: A Mechanomyographic Investigation. Muscle Nerve, 25: 505-12.

Awang, M.S, 2007. Nerve conduction study of healthy Asian Malays: The influence of age on median, ulnar, and sural nerves, Med. Sci. Monit., 13(7): CR330-332.

Bolton, C.F., Sawa, G.M., Carter, K. 1981. The effects of temperature on human compound action potentials.J. Neur. Neurosurg. Psych., 44: 407-13.

Brody, H. D. 1955. Organization of the cerebral cortex. III. A study of aging in the human cerebral cortex. J. Comp. Neurol. 102: 511516.
Brody, H. D. 1970. Structural changes in the aging nervous system. Interdisciplinary Topics in Gerontology. 7: 9-21.

Buchthal, F., Rosenfalck, A., Trojaborg, W. 1974. Electrophysiological findings in entrapment of the median nerve at wrist and elbow. $J$. Neur. Neurosurg. Psych., 37: 340-360.

Buchthal, F., Rosenfalck, A. 1971. Sensory conduction from digit to palm and from palm to wrist in the carpal tunnel syn.drome. $J$. Neur. Neurosurg. Psych., 34: 243-252.

Buschbacher, R.M., Koch, J. 1999. Race effect on nerve conduction studies: a comparison between 50 blacks and 50 whites. Arch. Phys. Med. Rehabil., 80: 536-539.

Campbell, M.J., McComas, A.J., Petito, F. 1973. Physiological changes in ageing muscles. $J$. Neur. Neurosurg. Psych., 36: 174-82.

Cannon, L.J., Bernacki, E.J., Walter, S.D. 1981. Personal and occupational factors associated with carpal tunnel syndrome. J. Occup. Med. 23: $255-258$.

Cunningham, D.A., Morrison, D., Rice, C.L., Cooke, C. 1987. Ageing and isokinetic plantar flexion. Eur. J. Appl. Physiol. 56: 24-29.

Davies, C.T.M., Thomas, D.O., White, M.J. 1986. Mechanical properties of young and elderly human muscle. Acta. Med. Scand., 711: 219226.

de Jesus, P.V., Hausmanowa-Petrusewicz, I., Baschi, R.L. 1973. The effect of cold on nerve conduction of human slow and fast nerve fibers. Neurology, 23:1182-1189.

Eklund, G. 1975. A new electrodiagnostic procedure for measuring sensory nerve conduction across the carpal tunnel. Upsala J. Med. Sci., 80: 63-64.

Falco, F.J., Hennessey, W.J., Braddom, R.L., Goldberg, G. 1992. Standardized nerve conduction studies in the upper limb of the healthy elderly. Am. J. Phys. Med. Rehabil., 71(5): 263-71.

Flack, B., Stålberg, E., Bischoff, C. 1994. Sensory nerve conduction studies with surface electrodes. Methods In: Clinical Neurophysiology. 5: 1-20.

Greathouse, D.G. 1989. Electrophysiologic Responses of Human Sural Nerve to Temperature. Physical Therapy 69(11): 3442.

Grimby, G., Aniansson, A., Zetterberg, C., Saltin, B. 1984. Is there a change in relative muscle fiber composition with age? Clin. Physiol., 4: 189-194 
Haug, H. 1984 Macroscopic and microscopic morphometry of the human brain and cortex. A survey in the light of new results. Brain Pathology. 1: 123-149.

Haug, H. 1985. Are neurons of the human cerebral cortex really lost during aging? A morphometric examination. In Senile dementia of the Alzheimer type (edited by TABER, J. \& GISPEN, W.) pp. 150-156. Berlin:Springer-Verlag.

Haug, H., Kuhl, S., Mecke, E., Sass, N. \& Wasner, K. 1984. The significance of morphometric procedures in the investigation of age changes in cytoarchitectonic structures of human brain. J. fur Hirnforschung, 25: 353374.

Henriksen, J.D. 1956. Conduction Velocity of Motor Nerves in Normal Subjects and Patients with Neuromuscular Disorders. Master's Thesis. Minneapolis, MN, University of Minnesota.

Jackson, D.A.; Clifford, J.C. 1989. Electrodiagnosis of mild carpal tunnel syndrome. Arch. Phys. Med. Rehabil. 70: 199-204.

Kallman, D.A., Plato, C.C., Tobin, J.D. 1990. The role of muscle loss in age-related decline in grip strength: cross-sectional and longitudinal perspectives. J. Geront., 45: M82-M88.

Lexell, J., Taylor, C.C., Sjostrom, M. 1988. What is the cause of the ageing atrophy? Total number, size and proportion of different fiber types studied in whole vastus lateralis muscle from 15- to 83-year-old men. J. Neurol. Sci., 84: 275-294.

Loong, S.C., Seah, C.S. 1971. Comparison of median and ulnar sensory nerve action potentials in the diagnosis of the carpal tunnel syndrome. J. Neur. Neurosurg. Psych., 34: 750-754.

Ludin, H.P., Beyeler, F. 1977. Temperature dependence of normal sensory nerve action potentials. J. Neur. Neurosurg. Psych., 216: 173-180.

McDonagh, M.J.N., White, M.J., Davies, C.T.M.1984. Different effects of aging on the mechanical properties of human arm and leg muscles. Geront., 30: 49-54.

McLeod, J.G. 1966. Digital nerve conduction in the carpal tunnel syndrome after mechanical stimulation of the finger. J. Neur. Neurosurg. Psych., 29: 12-22.

Mills, KR. 1985. Orthodromic sensory action potentials from palmar stimulation in the diagnosis of carpal tunnel syndrome. J. Neur. Neurosurg. Psych., 48: 250-255.

Monga, T.N., Shanks, G.L., Poole, B.J. 1985. Sensory palmar stimulation in the diagnosis of carpal tunnel syndrome. Arch. Phys. Med. Rehabil., 66: 598-600.

Oh, S.J. 1984. Clinical Electromyography and Nerve Conduction Studies. Baltimore, MD, University Park Press.

Peters, A 2002, The effects of normal aging on myelin and nerve fibers: A review. Journal of Neurocytology. 31: 581-593

Peters, A., Morrison, J. H., Rosene, D. L. \&Hyman, B. T. 1998 Are neurons lost from the primate cerebral cortex during aging? Cerebral Cortex 8: 295-300.

Porter, M.M., Vandervoort, A.A., Lexell, J. 1995. Aging of human muscle: structure, function and adaptability. Scand. J. Med. Sci. Sports, 5: $129-142$.

Rabben, O.K. 1995. Sensory nerve conduction studies in children. Age-related changes of conduction velocities. Neuropediatrics. 26(1): 26-32.

Rivner, M.H., Swift, T.R., Malik, K. 2001. Influence of age and height on nerve conduction. Muscle Nerve, 24(9): 1134-41.

Roos, M.R., Rice, C.L., Vandervoort, A.A. 1997. Age-related changes in motor unit function. Muscle Nerve, 20: 679-90.

Robinson, L.R., Rubner, D.E., Wahl, P.W., et al. 1993. Influences of height and gender on normal nerve conduction studies. Arch. Phys. Med. Rehabil., 74:1134-8.

Saeed, S. Akram. M. 2008. Impact of anthropometric measures on sural nerve conduction in healthy subjects. J. Ayub. Med. Coll. Abbottabad, 20(4): 112-114.

Salerno, D.F., Werner, R.A., Albers, J.W. 1999. Reliability of nerve conduction studies among active workers. Muscle Nerve, 22: 1372-79.

Silverstein, B.A., Fine, L.J., Armstrong, T.A. 1987. Occupational factors and carpal tunnel syndrome. Am. J. Indust. Med., 11: 343-358.

Soudmand, R., Ward, L.C., Swift, T.R.1982. Effect of height on nerve conduction velocity. Neurology. 32(4): 407-10.

Stalberg, E., Flack, B. 1993. Clinical motor nerve conduction studies. Methods. In: Clinical Neurophysiology, 4: 61-80.

Stetson, D.S 1982. Effects of age, sex, and anthropometric factors on nerve conduction 
measures, Muscle \& Nerve 15(10): 10951104.

Tackmann, W, Keaser, He, Magun, H.G. 1981. Comparison of orthodromic and antidromic sensory nerve conduction velocity measurements in the carpal tunnel syndrome. J. Neur. Neurosurg. Psych., 224: 257-266.

Terry, R. D., Deteresa, R. \& Hansen, L. A. 1987 Neocortical cell counts in normal human adult aging. Annals of Neurology, 21: 530539.

Thakur, D. 2010. Nerve Conduction Study in Healthy Individuals: a Gender Based Study, Health Renaissance, 8 (3); 169-175.

Thakur, D. 2011. Influence of height on the nerve conduction study parameters of the peripheral nerves, J. Clin. Diag. Res. 5(2): 260-263.
Tomlinson, B.E., Irving, D. 1977. The numbers of limb motor neurons in the human lumbosacral cord throughout life. J. Neurol. Sci. 34: 213-219.

Tong, H.C., Werner, R.A., Franblau, A. 2004. Effect of aging on sensory nerve conduction study parameters. Muscle Nerve, 29(5): 71620.

Wieslander, G., Norback, D., Ljuhlin, C.l.C. 1989. Carpal tunnel syndrome (CTS) and exposure to vibration, repetitive wrist movements, and heavy manual work: a case-refinement study. Brit. Lndus. Med., 46: 43-47.

Young, A., Stokes, M., Crowe, M. 1984. Size and strength of the quadriceps muscles of old and young women. Eur. J. Clin. Invest., 14: 282287. 